\title{
Effect of ductile layers in mechanical behaviour of TiAlN thin coatings
}

\author{
J.M. Castanho, M.T. Vieira* \\ ICEMS, Mechanical Engineering Department, University of Coimbra, Coimbra 3030, Portugal
}

\begin{abstract}
TiAlN is a very popular coating for high-speed cutting manufacturing processes. Nevertheless, it is important to develop research work concerning the durability of this kind of coatings. Design and producing coatings based on TiAlN with interlayers of metal with low elastic modulus, like $\mathrm{Al}$, Ti or $\mathrm{Cu}$ may contribute to improvement of cutting efficiency. Interlayers with $80 \mathrm{~nm}$ of thickness, in alternateness with TiAlN with different periods were sputtered. In general, the hardness and Young's modulus of the multilayer coatings had slight smaller values, but similar relations of $H / E$ in monolithic TiAlN coatings. However, in multilayers based on $\mathrm{Al}$ and $\mathrm{Cu}$ there is an important decrease in the $H^{2} / E$ ratio. Moreover, the adhesion $\mathrm{Lc}_{2}$ values of scratch test show an important increase, particularly in $\mathrm{Al}$ and Ti interlayers, which can be considered as an important contribution in the improvement of the cutting performance of TiAlN-based coatings.
\end{abstract}

(C) 2003 Published by Elsevier Science B.V.

Keywords: Multilayer coatings; Adhesion; Internal stress; Mechanical properties; TiAlN; Hard coatings

\section{Introduction}

Different kinds of coatings have been studied and developed using several types of deposition processes. The tribological behaviour of these coatings in heavy-duty mechanical components such as twist drills, cutting tools, dies, etc., assure a relevant position in industry. The capacity of some coatings to be used in severe working conditions can be attributed to the high hardness and wear resistance, as well as a good oxidation and corrosion resistance. The industry, with implications in higher machining speeds and environment friendly technologies, expected higher temperature oxidation resistant materials [1]. TiAlN thin films are now commercially available as wear resistant coatings for high-speed machining, due to their high hardness, excellent oxidation and corrosion resistance. Since the beginning of the production of the coatings to be used in cutting applications, TiN films were chosen due to their good mechanical properties. However, the loose of hardness and oxidation of these coatings, at cutting temperatures, refrained the cutting speed. The presence of $\mathrm{Al}$ in TiAlN coatings overcomes the oxidation problems due to the presence of a superficial layer of $\mathrm{Al}_{2} \mathrm{O}_{3}$ formed at high temperatures [2-8].

The possibility to sputter coatings with several layers of different materials opens new directions in the production of structural coatings. The main purpose of this work is

\footnotetext{
* Corresponding author.

E-mail address: teresa.vieira@mail.dem.uc.pt (M.T. Vieira).
}

to improve the durability of these coatings by placing into TiAlN layer interlayers of ductile metal, without causing a disruption in the mechanical properties of TiAlN. Such interlayers can enhance the "resilience" and adhesion of the coatings. Hence, three ductile metals were used as interlayers: aluminium, titanium and copper. Aluminium and titanium have different type of structures, A1-f.c.c. and A3-h.c.p. and bulk Young's modulus of 69 and $116 \mathrm{GPa}$, respectively, but they have reactivity to nitrogen. Due to the ductility and non-reactivity to nitrogen, copper (A1-f.c.c.) was also selected as metal interlayer. Additionally, if the first layer of the ceramic coating could promote a gradient of the chemical and mechanical properties between the substrate and the TiAlN layer, the possibility to develop a superior coating increases.

\section{Experimental details}

Multilayer coatings were deposited on heat-treated (quenching and tempering), polished $\left(R_{\mathrm{a}}<0.02 \mu \mathrm{m}\right)$ high-speed steel M2 (AISI) with hardness of $9 \mathrm{GPa}$, by a two d.c. magnetron semi-industrial sputtering prototype (HARTEC). A titanium aluminide target (51.9 at.\% of Ti, 40.6 at.\% of $\mathrm{Al}, 1.4$ at.\% of $\mathrm{Cr}$ and 6.1 at.\% of $\mathrm{O}$ ) and $\mathrm{N}_{2}$ as reactive gas with a partial pressure of $\mathrm{N}_{2} / \mathrm{Ar}$ ratio of 0.3 was used to deposit the ceramic TiAlN layers, and a pure metal (aluminium, titanium or copper) target to sputter the interlayers. The deposition pressure, substrate bias and substrate-to-target distance were kept constant at $0.3 \mathrm{~Pa}$, 
$-70 \mathrm{~V}$ and $65 \mathrm{~mm}$, respectively. In order to create a nitrogen gradient in the first layer, all coatings were deposited with an initial layer of TiAl (without reactive gas) then the flow of nitrogen was increased up to the ultimate partial pressure. The thickness of the metal interlayers was kept constant and equal to $80 \mathrm{~nm}$. The total thickness of the coatings was $3.5 \mu \mathrm{m}$. The deposition rates of both ceramic and metal films were determined in a previous study. Prior to deposition, all the substrates were heated and etched $(-70 \mathrm{~V})$ for $900 \mathrm{~s}$ at $0.15 \mathrm{~Pa}$ in argon atmosphere. The temperature achieved in the substrates was lower than $500^{\circ} \mathrm{C}$.

The chemical composition of the coatings was evaluated by electron probe microanalysis (Cameca SX 50). The as-deposited coating structures were analysed by X-ray diffraction equipment (Philips X'Pert) using Co $\mathrm{K} \alpha$ radiation, in a $\theta-2 \theta$ Bragg-Brentano mode. The hardness and the Young's modulus of the coatings were evaluated by depth-sensing-indentation hardness equipment (Fischerscope H100) with a Vickers tip using loads of 50, 100, 200, 300, 500 and $1000 \mathrm{mN}$ and 10 measurements were performed in each load. The loads of $50 \mathrm{mN}$ were used to determine the hardness of the coatings due to the low penetration depth $(<0.3 \mu \mathrm{m})$ which apart any contribution of the substrate. The entire set of load was necessary to evaluate the Young's modulus using the Gao et al.'s method [9]. The residual stresses of the coatings were determined by beam bending analysis using the Stoney equation [10]. The adhesion $\left(\mathrm{Lc}_{2}\right)$ of the coatings was evaluated by a commercial CSEM Revetest scratch-tester in the range of $0-70 \mathrm{~N}$.

\section{Results and discussion}

\subsection{TiAlN monolithic coatings}

The morphology of the monolithic coating is dense and columnar as illustrated in Fig. 1. This coating has a chemical composition in accordance with $\left(\mathrm{Ti}_{0.46} \mathrm{Al}_{0.54}\right)_{0.96} \mathrm{~N}$ (although the oxygen content in the coating was determined, it was not significant for this study) and its mechanical characteristics are presented in Table 1.

The structure of TiAlN coating is an f.c.c. TiN (1 111$)$ oriented structure (JCPDS-ICDD 87-0633) with a lattice parameter of $0.422 \pm 0.005 \mathrm{~nm}$. Having in mind the chemical

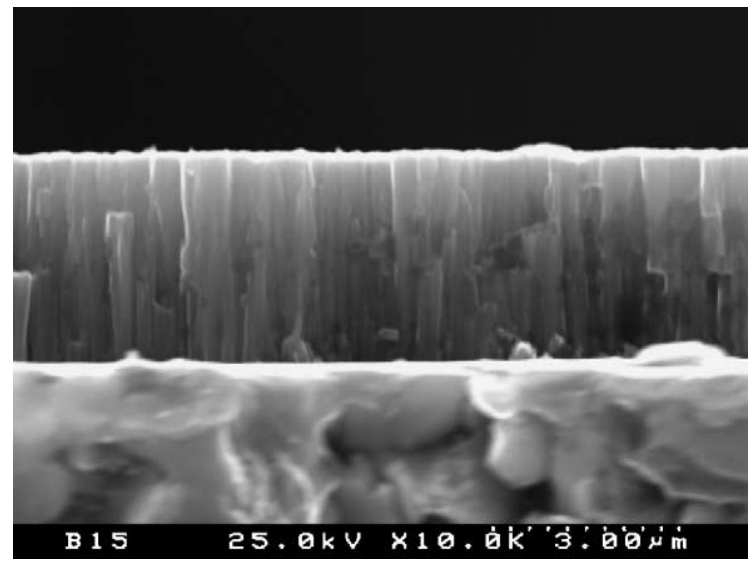

Fig. 1. Cross-sectional view of TiAlN monolithic coating.

composition of the coatings, the aluminium atoms substitute the titanium atoms in the lattice. The lattice parameters of the TiAlN coatings, with similar chemical composition, found in the literature are slightly smaller, in the range $0.417-0.420 \mathrm{~nm}[1,3,4,11-14]$. TiAlN coating present an excess of aluminium relative to the chemical composition of the target, which can be due to the higher deposition rate of aluminium relative to titanium.

The hardness of $\left(\mathrm{Ti}_{0.46} \mathrm{Al}_{0.54}\right)_{0.96} \mathrm{~N}$ coating is slightly higher than the values found in the literature for similar chemical composition $[1,8,13,15]$, but is lower than the other coatings of multicomponent nitrides of refractory metals, such as W-Ti-N [16,17]. Moreover, the $H / E$ values are similar to TiN and lower than other transition metal nitrides $\left(\mathrm{M}_{1}-\mathrm{M}_{2}-\mathrm{N}\right)$ [18]. TiAlN has a compressive residual stress of $1.8 \mathrm{GPa}$ and exhibit good adhesion.

\subsection{TiAlN multilayer coatings}

\subsubsection{Aluminium interlayers}

The mechanical properties of TiAlN coatings with aluminium interlayers are summarised in Table 2. The presence, in the coating, of one aluminium interlayer with $80 \mathrm{~nm}$ of thickness is enough to reduce in $25 \%$ the hardness of the coatings. In addition, as the number of the interlayers increases the hardness of the coating decreases, this fact is particularly relevant in the coatings with 11 or more layers. In general, the Young's modulus of the multilayer coatings

Table 1

Characterisation of TiAlN monolithic coating

\begin{tabular}{|c|c|c|c|c|c|}
\hline \multicolumn{4}{|c|}{ Chemical composition (at.\%) } & \multirow{2}{*}{\multicolumn{2}{|c|}{ Structure }} \\
\hline $\mathrm{Ti}$ & $\mathrm{Al}$ & \multicolumn{2}{|l|}{$\mathrm{N}$} & & \\
\hline $21.9 \pm 0.2$ & $25.6 \pm 0.1$ & \multicolumn{2}{|c|}{$50.1 \pm 0.3$} & & \\
\hline \multicolumn{4}{|c|}{ Mechanical characteristics } & \multicolumn{2}{|c|}{ B1-TiN } \\
\hline $\mathrm{Lc}_{2}(\mathrm{~N})$ & $H_{\mathrm{V} 50 \mathrm{mN}}(\mathrm{GPa})$ & $E(\mathrm{GPa})$ & $\sigma_{\text {resid }}(\mathrm{GPa})$ & $H / E$ & $H^{2} / E$ \\
\hline $42 \pm 1.5$ & $30 \pm 3$ & $632 \pm 8$ & $-1.8 \pm 0.5$ & 0.05 & 1.42 \\
\hline
\end{tabular}


Table 2

Mechanical properties of multilayer coatings with aluminium interlayers

\begin{tabular}{|c|c|c|c|c|c|c|}
\hline Number of layers/interlayers & $\mathrm{Lc}_{2}(\mathrm{~N})$ & $H_{\mathrm{V} 50 \mathrm{mN}}(\mathrm{GPa})$ & $E(\mathrm{GPa})$ & $\sigma_{\text {resid }}(\mathrm{GPa})$ & $H / E$ & $H^{2} / E$ \\
\hline $3 / 1$ & $34 \pm 4$ & $23 \pm 2$ & $460 \pm 11$ & $-1.6 \pm 0.1$ & 0.05 & 1.15 \\
\hline $7 / 3$ & $>70$ & $24 \pm 1$ & $620 \pm 20$ & $-1.9 \pm 0.2$ & 0.04 & 0.92 \\
\hline $11 / 5$ & $48 \pm 3$ & $19 \pm 1$ & $361 \pm 4$ & $-2.1 \pm 0.2$ & 0.05 & 0.95 \\
\hline $15 / 7$ & $58 \pm 5$ & $18 \pm 1$ & $363 \pm 4$ & $-2.5 \pm 0.7$ & 0.05 & 0.87 \\
\hline
\end{tabular}

suffers a decrease relative to the monolithic TiAlN coatings. However, an unexpected high Young's modulus value is attained in the coatings with seven layers. The $H / E$ ratio is independent of the number of aluminium interlayers presented in the coating. However, the $H^{2} / E$ decreases as the number of layers increases. The compressive residual stresses of the coatings increase, from -1.6 to $-2.5 \mathrm{GPa}$, as the number of the layers increases. This fact can be understood by the presence of interface mismatches and thermal stresses induced by materials with very different thermal expansion coefficients $\left(\mathrm{Ti}_{0.46} \mathrm{Al}_{0.54}\right)_{0.96} \mathrm{~N}$ and $\mathrm{Al}$.

The adhesion of the coatings increases relative to the TiAlN monolithic coating achieving values higher than $70 \mathrm{~N}$ in coatings with seven layers, such increase can result from the accommodation, due to the presence of aluminium, of the stresses induced by the indenter. Fig. 2 shows a cross-sectional view of an indentation channel in which is clearly revealed the good adhesion of the coating to substrate and the deformation caused by the indenter. Fig. 3 presents the end of the indentation channel, where is clearly shown the delamination of the coating, and that only in a few zones has reached the substrate.

\subsubsection{Titanium interlayers}

The morphology of TiAlN multilayer coatings with titanium interlayers is also columnar, as is shown in Fig. 4. The coating with $n$ layers reveals a continuous columnar morphology, with no evidence of the interlayers (Fig. 5).

In general, the hardness of multilayer coatings is in the range of the TiAlN monolithic coating (Table 3). Further-

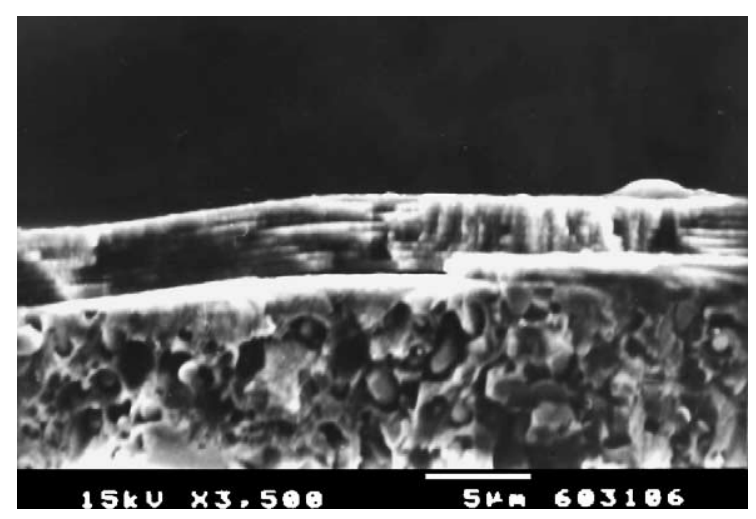

Fig. 2. Cross-sectional view of the deformation of TiAlN 15-layered coating due to the scratch test.

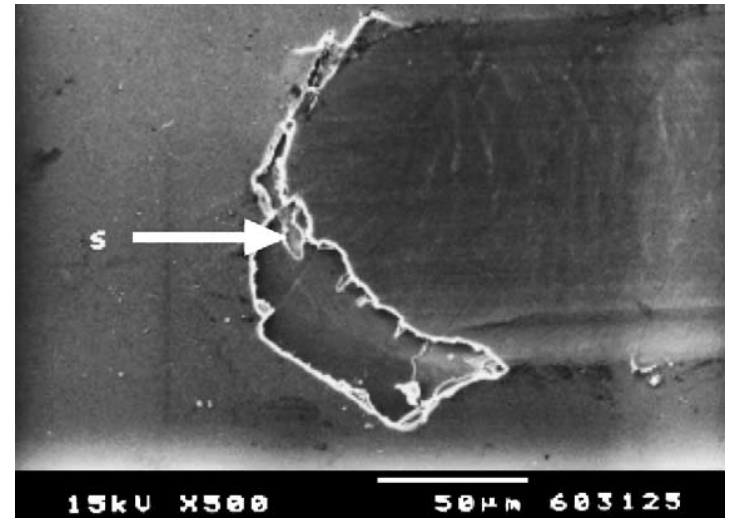

Fig. 3. Final of the indentation channel of TiAlN 15-layered coating (s: substrate).

more, the coatings with $n$ layers are only softer than the others.

The Young's modulus of the coatings follows the same tendency as their hardness. The values of the $H / E$ ratio are similar to the other multilayer coatings studied. However, in general the $H^{2} / E$ ratio has values equal to or higher than the monolithic coating. Concerning the residual stress, the presence of one titanium interlayer is enough to reduce to half the internal stress of the TiAlN coatings. However, as the number of layers increases the compressive residual stress also increases, reaching the value of the TiAlN monolithic film in the coatings with more than 11 layers. This behaviour is very

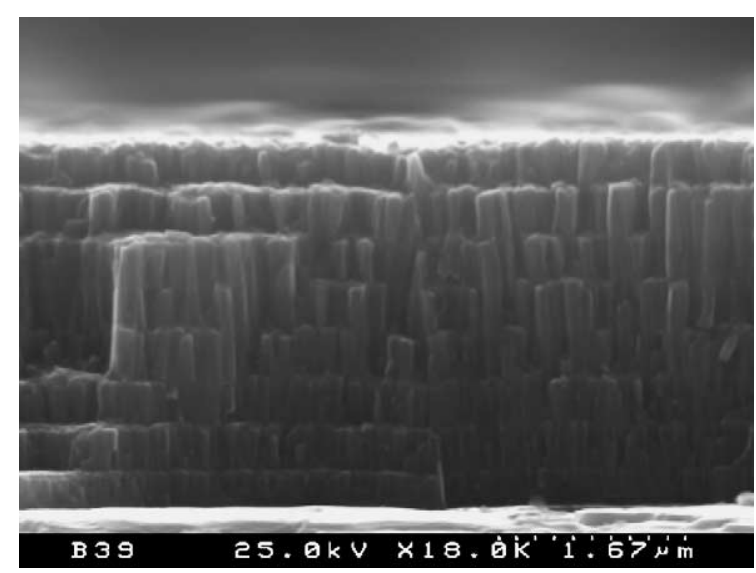

Fig. 4. Cross-sectional view of TiAlN 15-layered coating with titanium interlayers. 
Table 3

Mechanical properties of multilayer coatings with titanium interlayers

\begin{tabular}{|c|c|c|c|c|c|c|}
\hline Number of layers/interlayers & $\mathrm{Lc}_{2}(\mathrm{~N})$ & $H_{\mathrm{V} 50 \mathrm{mN}}(\mathrm{GPa})$ & $E(\mathrm{GPa})$ & $\sigma_{\text {resid }}(\mathrm{GPa})$ & $H / E$ & $H^{2} / E$ \\
\hline $3 / 1$ & $54 \pm 15$ & $24 \pm 2$ & $550 \pm 8$ & $-0.93 \pm 0.04$ & 0.05 & 1.14 \\
\hline $7 / 3$ & $67 \pm 1$ & $32 \pm 2$ & $600 \pm 20$ & $-1.2 \pm 0.1$ & 0.05 & 1.71 \\
\hline $11 / 5$ & $68 \pm 3$ & $32 \pm 2$ & $623 \pm 12$ & $-1.7 \pm 0.1$ & 0.05 & 1.64 \\
\hline $15 / 7$ & $66 \pm 3$ & $28 \pm 2$ & $559 \pm 12$ & $-1.9 \pm 0.1$ & 0.05 & 1.40 \\
\hline$n(\sim 340)$ & $32 \pm 4$ & $22 \pm 3$ & $360 \pm 20$ & $-2.5 \pm 0.1$ & 0.06 & 1.32 \\
\hline
\end{tabular}

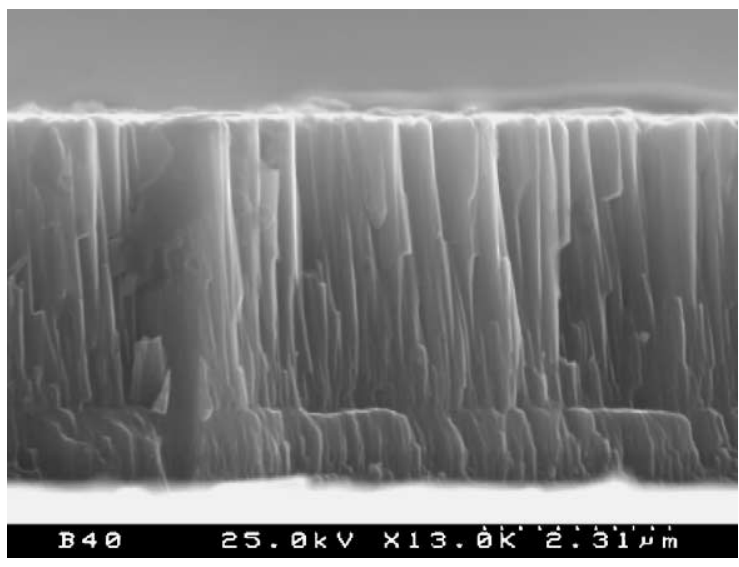

Fig. 5. Cross-sectional view of TiAlN $n$-layered coating with titanium interlayers.

similar to the one presented by the TiAlN multilayer coatings with aluminium interlayers. All films presented high adhesion values, and only in small zones the failures reach the substrate, as is shown in Fig. 6. The coatings with $n$ layers exhibit low adhesion and catastrophic failures, which easily reach the substrate (Fig. 7).

\subsubsection{Copper interlayers}

The morphology of TiAlN coatings with copper interlayers is also dense and columnar and the interfaces are well defined, Fig. 8. The introduction of copper interlayers results in a decrease of $25 \%$ in the hardness of the coatings, as in the films with aluminium interlayers. How-

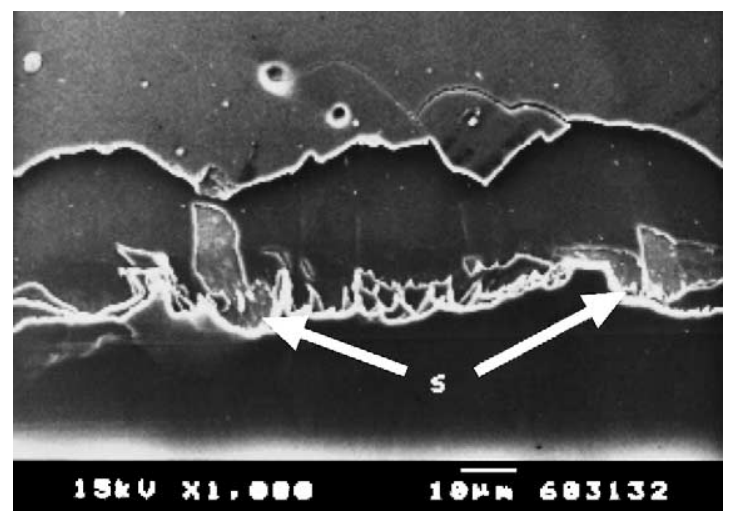

Fig. 6. Failure of the TiAlN 11-layered coating with titanium interlayers (s: substrate).

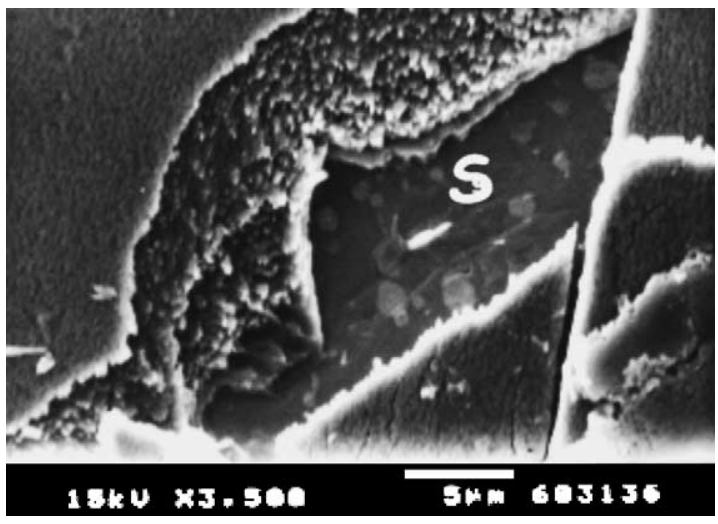

Fig. 7. Failure of the TiAlN n-layered coating with titanium interlayers (s: substrate).

ever, such decrease is more severe in the coatings with the highest number of layers (Table 4). The Young's modulus of the films decrease as the number of layers increases, reaching a value very similar to the steel substrates in the coatings with $n$ layers. The factor $H / E$ decreases as the number of layers increase. The $H^{2} / E$ ratio has a very significant decrease as the number of interlayers increases. This decrease is particularly evident in the coatings with 15 layers or more. In general, the compressive residual stress of these coatings decreases, especially for coatings with the highest number of interlayers, being particularly relevant with the values presented by coatings with 15 or more layers. The adhesion of the coatings with one copper interlayer is similar to the adhesion presented by the TiAlN monolithic coating, and as

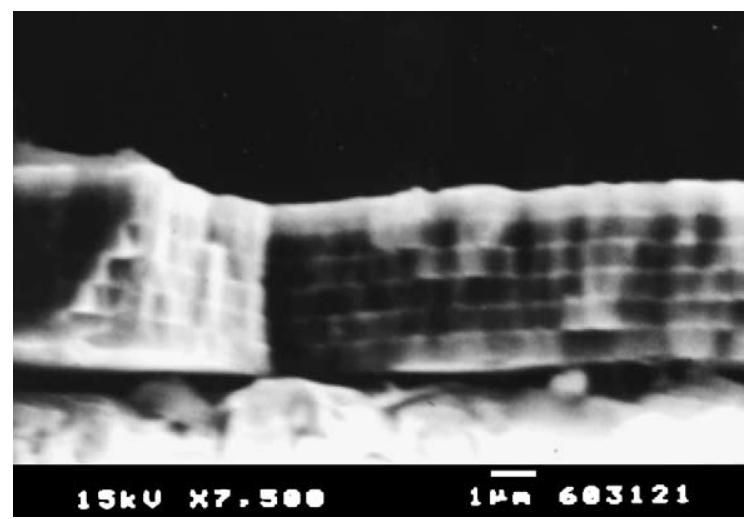

Fig. 8. Cross-sectional view of TiAlN 11-layered coating with copper interlayers. 
Table 4

Mechanical properties of multilayer coatings with copper interlayers

\begin{tabular}{llllrr}
\hline Number of layers/interlayers & $\mathrm{Lc}_{2}(\mathrm{~N})$ & $H_{\mathrm{V} 50 \mathrm{mN}}(\mathrm{GPa})$ & $E(\mathrm{GPa})$ & $\sigma_{\text {resid }}(\mathrm{GPa})$ & $H / E$ \\
\hline $3 / 1$ & $41 \pm 4$ & $22 \pm 2$ & $552 \pm 5$ & $-1.4 \pm 0.1$ & 0.04 \\
$7 / 3$ & $33 \pm 2$ & $24 \pm 3$ & $496 \pm 7$ & $-1.5 \pm 0.1$ & 0.05 \\
$11 / 5$ & $23 \pm 5$ & $16 \pm 2$ & $437 \pm 6$ & $-1.3 \pm 0.1$ & 0.04 \\
$15 / 7$ & $25 \pm 1$ & $8.7 \pm 0.5$ & $329 \pm 7$ & $-0.54 \pm 0.03$ & 0.03 \\
$n(\sim 340)$ & $11 \pm 1$ & $6.9 \pm 0.4$ & $238 \pm 8$ & $0.17 \pm 0.01$ & 0.03 \\
\hline
\end{tabular}

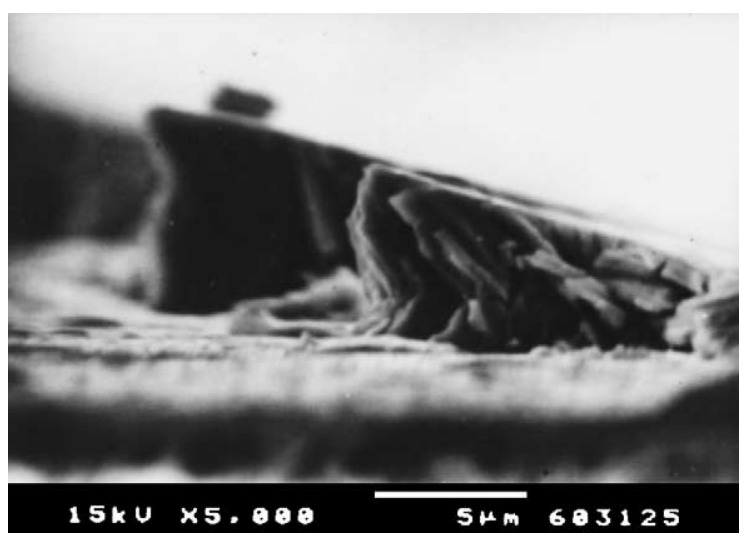

Fig. 9. Delamination in the indentation channel.

the number of layers increases the lower is the adhesion of the films. The failures presented by the coatings are catastrophic with strong delamination in the indentation channel, Fig. 9.

\section{Discussion}

The structure of TiAlN monolithic coating is a TiN-f.c.c. with a lattice parameter of $0.422 \mathrm{~nm}$, this is similar to the structure presented by aluminium and copper. However, the difference in the lattice parameter between the TiAlN $(0.422 \mathrm{~nm})$ and aluminium $(0.405 \mathrm{~nm})$ is smaller than copper $(0.361 \mathrm{~nm})$. So, a more coherent interface between TiAlN and aluminium probably can be found, resulting in better mechanical properties in the coatings with aluminium interlayers. Besides, the structure of titanium is A3-h.c.p., which is incoherent with TiAlN structure, but the coatings with titanium interlayers maintain the mechanical properties of monolithic TiAlN coating. Therefore, the structure cannot be the major reason to explain the performance differences of the coatings with different metal interlayers. Aluminium and titanium have similar atomic radii, higher than the radius of copper. Therefore, it is possible that the growth of the interlayers could be ruled by atomic substitution and the chemical affinity surpasses the structural affinity of each metal. Therefore, titanium must have better affinity to $\left(\mathrm{Ti}_{0.46} \mathrm{Al}_{0.54}\right)_{0.96} \mathrm{~N}$ layers than aluminium. Other reason is that although the flow of nitrogen vanishes during the deposition of the metal interlayers, it is possible that residual nitrogen is still present in the deposition chamber and reacts with the ductile metal and the earliest atomic layers of the interlayers are constituted by metal nitrides. Aluminium nitride has a B4-h.c.p. structure while titanium nitride has a B1-f.c.c. structure, which is very similar to the one presented by TiAlN.

All coatings exhibit a dense columnar morphology and no difference can be observed in the multilayer coatings with different metal interlayers. The multilayer coatings with a large number of layers (340) show a columnar and continuous morphology with no evidence of the interlayers, which is due to the nanometre character of the layers.

The presence of titanium interlayers does not decrease the hardness of the multilayer coating relative to the monolithic TiAlN coating. In fact, the presence of the titanium interlayer is not "seen" by the indenter, which is probably due to the coherency of the interfaces and the presence of nitrogen in the interlayer. Moreover, the aluminium and copper interlayers contribute in the same way to the decrease of the hardness of the multilayer coatings. The presence of one metal interlayer is enough to decrease the hardness of the coating.

The elastic response of the multilayer coatings is affected by the presence of the interlayers, especially in the coatings with a large number of layers and particularly in the coatings with aluminium and copper where the interface strength is weaker. However, while in the multilayer coatings with copper interlayers the Young's modulus decrease as the number of layers increase, the coatings with aluminium and titanium presented an unexpected high value of Young's modulus in the coatings with seven and 11 layers, respectively. The $H / E$ and $H^{2} / E$ ratios are claimed by different authors [18] as a method to evaluate the tribological and "resilience" performance of the coatings. Concerning the multilayer films studied, it can be observed that the presence of ductile layers does not contribute to an important modification of $H / E$ in comparison to the monolithic coatings. Furthermore, the values obtained are similar to $\mathrm{TiN}$ and $\mathrm{CrN}$ [18]. Nevertheless, the presence of a titanium interlayer contributes to an increase of $H^{2} / E$ ratio, in particular to seven and 11 layers coatings. This may be a sign that this kind of coatings must not be used when the substrate is deformable. However, the results of the scratch-test seem to follow the $H^{2} / E$ factor indication. In fact, the coatings with aluminium and titanium interlayers show a better behaviour to deformation.

The introduction of the metal interlayers (like aluminium and titanium) contributes to the increase in the adhesion of 
the multilayer coatings to substrate. In fact, although the thickness of the interlayers is $80 \mathrm{~nm}$, it is enough to promote the deformability of the coating without fracture. The adhesion between the TiAlN and the titanium interlayers is stronger than the adhesion with aluminium interlayers. Conversely, the copper interlayers reduce drastically the adhesion of the coatings, which is due to the high ductility of the copper and the TiAlN layers easily breaks.

Only a single interlayer is enough to decrease the internal stresses of the multilayer coating relative to the TiAlN monolithic coating, especially in the coatings with titanium interlayers. In the multilayer coatings with aluminium and titanium interlayers, as the number of interlayers increases more compressive stresses are installed in the film. The coatings with copper interlayers present an opposite behaviour. Such fact can be due to the non-reactivity of copper to nitrogen, and the stresses induced by the growing process can be released by the presence of these metal interlayers.

\section{Conclusions}

All coatings exhibit a dense columnar morphology whatever are the metal of interlayers.

The presence of ductile metal interlayers may reduce the hardness and the Young's modulus of coatings, but not the ratio $H / E$. The $H^{2} / E$ is affected by the presence of the metal interlayers. The $\mathrm{Al}$ and $\mathrm{Cu}$ interlayers reduce the ratio compared to the monolithic coatings.

Titanium and aluminium interlayers increase the compressive residual stresses of the coatings while copper interlayers decrease them. The presence of only one ductile metal interlayers may reduce the residual stress of the coating. This behaviour is similar to the other transition metal nitrides multilayer coatings.

Titanium and aluminium interlayers increase significantly the adhesion of the coatings, but the copper interlayers decrease it.
Aluminium and titanium interlayers are more suitable to produce efficient multilayer coatings than the copper ones.

\section{References}

[1] L.P. Ward, K.N. Strafford, C. Subramanian, T.P. Wilks, J. Mater. Process. Technol. 56 (1996) 375-384.

[2] M. Pinkas, J. Pelleg, M.P. Daniel, Thin Solid Films 355-356 (1999) 380-384.

[3] T. Suzuki, D. Huang, Y. Ikuhara, Surf. Coat. Technol. 107 (1998) 41-47.

[4] M. Zhou, Y. Makino, M. Nose, K. Nogi, Thin Solid Films 339 (1999) 203-208.

[5] K.-D. Bouzakis, N. Vidakis, N. Michailidis, T. Leyendecker, G. Erkuens, G. Fuss, Surf. Coat. Technol. 120 (1999) 34-43.

[6] A. von Richthofen, R. Cremer, M. Witthaut, R. Domnick, D. Neuschütz, Thin Solid Films 312 (1998) 190-194.

[7] L. Cunha, M. Andritschky, L. Rebouta, R. Silva, Thin Solid Films 317 (1998) 351-355.

[8] C. Jiménez, C. Sanchéz-Fernández, C. Morant, J.M. Martínez-Duart, M. Fernández, J. Sánchez-Olías, J. Mater. Res. 14 (7) (1999) 28302837.

[9] H. Gao, C.H. Chiu, J. Lee, Int. J. Solids Struct. 29 (1992) 2471-2492.

[10] G.G. Stoney, Proc. R. Soc. London A 82 (1909) 172.

[11] H.A. Jehn, S. Hofmann, W.-D. Münz, Thin Solid Films 153 (1987) 45-53.

[12] G. Håkansson, J.-E. Sundgren, D. McIntyre, J.E. Greene, W.-D. Münz, Thin Solid Films 153 (1987) 55-65.

[13] C. Jarms, H.-R. Stock, P. Mayr, Surf. Coat. Technol. 108-109 (1998) 206-210.

[14] P. Panjan, B. Navinšek, M. Čekada, A. Zalar, Vacuum 53 (1999) 127-131.

[15] J. Musil, H. Hrubý, Thin Solid Films 365 (2000) 104-109.

[16] M.T. Vieira, C.M. Pereira, J.M. Castanho, Surf. Coat. Technol. 131 (2000) 417-421.

[17] J.M. Castanho, M.T. Vieira, Proceedings of the Seventh International Conference on Composites Engineering, Denver (EUA), 2000, pp. 105-106.

[18] A. Matthews, A. Leyland, Proceedings of the Seventh International Conference of the European Ceramic Society (EcerS'2001), Brugge, pp. $459-466$. 